\title{
VHF-electromagnetic evidence of the underlying pre-seismic critical stage
}

\author{
Panayiotis Kapiris $^{1}$, John Polygiannakis ${ }^{1}$, Athanassios Peratzakis ${ }^{1}$, Konstantinos Nomicos ${ }^{2}$, and Konstantinos Eftaxias ${ }^{1}$ \\ ${ }^{1}$ Department of Physics, University of Athens, Zografos, 15784 Athens, Greece \\ ${ }^{2}$ Technological Educational Institute of Athens, Egaleo, 12210, Greece
}

(Received February 20, 2002; Revised October 7, 2002; Accepted November 26, 2002)

Based on the study of pre-seismic very high frequency (VHF) and very low frequency (VLF) electromagnetic signals, we attempt to establish a set of necessary conditions referring to the underlying critical stage of the earthquake preparation process. This study combines concepts from spectral analysis associated with critical point hypothesis, results from laboratory experiments of rupture and seismological arguments. These conditions are fully satisfied in the case of the VLF-VHF pre-seismic signals associated with the Kozani-Grevena earthquake in Greece.

\section{Introduction}

VLF-VHF electromagnetic (EM) emissions have been detected a few days to a few hours prior to recent destructive earthquakes (EQs) in Greece (Eftaxias et al., 2000). We have shown that the features of these emissions are possibly correlated with the fault model characteristics suggested for each EQ (Eftaxias et al., 2001) and the degree of heterogeneity within the focal area (Eftaxias et al., 2000). Moreover, Eftaxias et al. (2002a) evaluated these signals in terms of their relation to EQs in comparison with laboratory measurements on rock samples carried out by Ohnaka and Mogi (1982). Thus, the idea that the detected pre-seismic EM anomalies are probably emitted from the focal area during micro-fracturing process appears reasonable.

We note that recently, Asada et al. (2001) have presented an attempt to delineate VLF ( $1 \sim 10 \mathrm{kHz})$ EM signals associated with EQs. The association of the VLF emissions with the occurrence of an EQ is inferred from (a) temporal correlation and (b) agreement between the direction of VLF signal arrival and that epicentre. The epicentre location before the EQ is very important. A guiding concept underlying their technique is that signals observed at a location in association with an EQ must come predominantly from a distinct direction, namely, that of the epicentre, while the signal sources of sferics are dispersed in azimuth when viewed from a single site.

However, more data on the presence of the VLF-VHF EM signatures of EQs need to be accumulated. There is accumulating evidence that large EQs are critical phenomenon (Sornette, 2000). Thus, combining concepts associated with critical point hypothesis, results from acoustic and EM laboratory measurements on rock samples and seismological arguments, we look for new EM conditions referring to the underlying last critical stage of the EQ preparation.

Copy right(c) The Society of Geomagnetism and Earth, Planetary and Space Sciences (SGEPSS); The Seismological Society of Japan; The Volcanological Society of Japan; The Geodetic Society of Japan; The Japanese Society for Planetary Sciences.

\section{Observation System}

Aiming at recording VLF-VHF EM precursors, since 1994 a station was installed at a mountainous site of Zante island $\left(37.76^{\circ} \mathrm{N}-20.76^{\circ} \mathrm{E}\right)$ in western Greece (Fig. 1) with the following configuration: (i) six loop antennas detecting the three components (EW, NS, and vertical) of the variations of the magnetic field at $3 \mathrm{kHz}$ and $10 \mathrm{kHz}$ respectively; and (ii) two vertical $\lambda / 2$ electric dipoles detecting the strength of the electric field at $41 \mathrm{MHz}$ and $54 \mathrm{MHz}$ respectively. The used VLF, VHF receivers are chosen to operate in their linear input region with a constant gain $G_{r}$. The output of each receiver is then filtered by a passive lowpass filter with cutoff frequency $1 \mathrm{~Hz}$ which defines the appropriate bandwidth for the used acquisition system. The bandpass gain up to $0.5 \mathrm{~Hz}$ is constant and equal to $G_{L P F} \approx 1$. The 3 and 10 $\mathrm{kHz}$ and 41 and $54 \mathrm{MHz}$ frequencies were selected to minimize the effects of the sources of cultural noise. All the EM time-series were sampled at $1 \mathrm{~Hz}$ (Nomikos and Vallianatos, 1998; Vallianatos and Nomikos, 1998; Eftaxias et al., 2000).

Such an experimental set-up helps to specify not only whether or not a single VLF or VHF EM disturbance is preseismic in itself, but also whether a combination of such disturbances at different frequency bands, could be characterized as pre-seismic according to accumulated laboratory and field experience (Eftaxias et al., 2000, 2002a).

\section{Electromagnetic Anomalies before the Kozani- Grevena Earthquake}

On May 13, 1995, at 08:47 UT, a magnitude $M_{S}(A T H)=$ 6.6 EQ struck the Kozani-Grevena (Fig. 1) in NW Greece $\left(40.2^{\circ} \mathrm{N}-21.7^{\circ} \mathrm{E}\right)$. VLF-VHF EM anomalies (EMAs) have been observed before this destructive EQ at Zante station $\left(37.76^{\circ} \mathrm{N}-20.76^{\circ} \mathrm{E}\right)$ with the following order: (i) a few tens of hours prior to the Kozani-Grevena EQ EMAs were recorded at $41 \mathrm{MHz}$ and $54 \mathrm{MHz}$ respectively, with increasing EM emission rate (Fig. 2(a)). These anomalies ceased approximately one hour before the EQ occurrence; (ii) very strong multi-peaked EM signals with sharp onsets and ends at $3 \mathrm{kHz}$ and $10 \mathrm{kHz}$, lasting about half hour, emerged ap- 


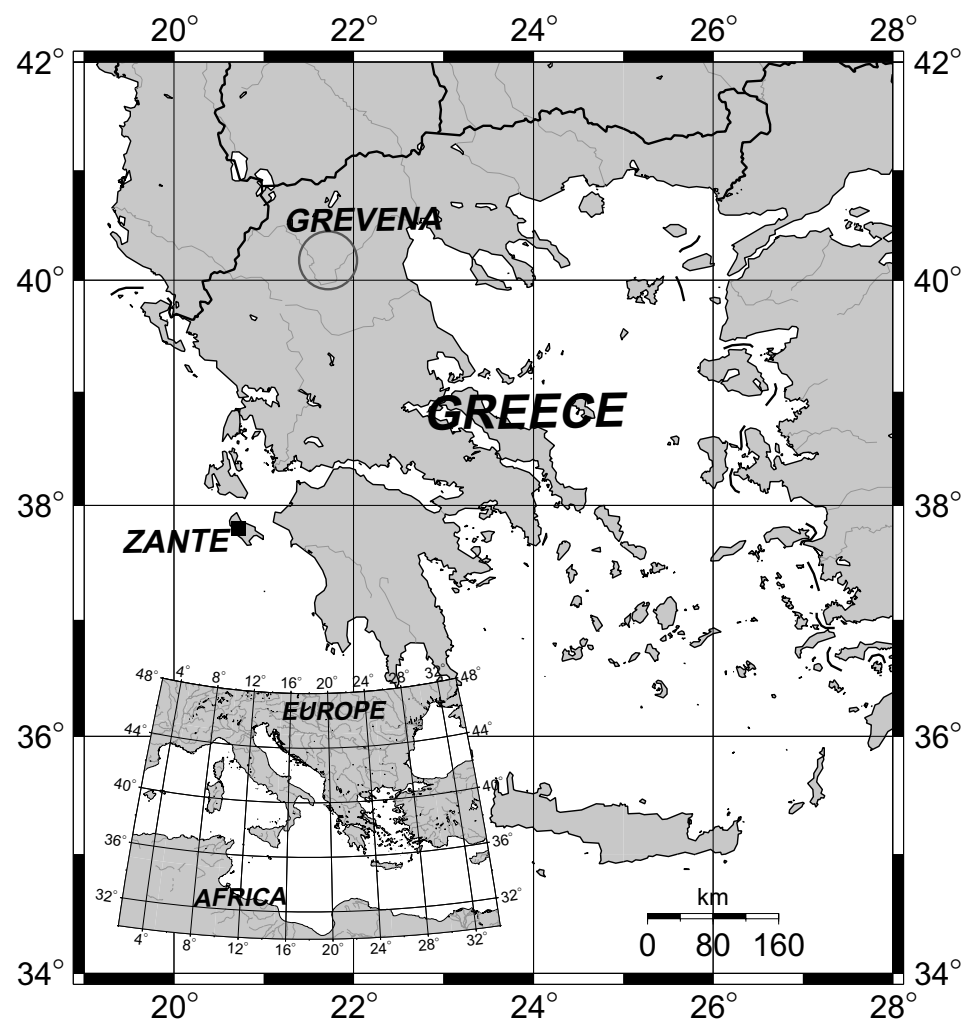

Fig. 1. A map of Greece demonstrating the location of Zante station and the epicentre of the Kozani-Grevena EQ.

proximately one and half hour before the EQ (Fig. 2(b)) (Eftaxias et al., 2000). We draw the attention at the almost simultaneous cessation of these EMAs at both $(\mathrm{kHz}$ and $\mathrm{MHz}$ ) frequency bands although they had very different onset times. Asada et al. (2001) suggest that the fact that the time of maximum VLF emission does not coincide with that of the main shock of the EQ must be considered to constitute an important element in the VLF emission mechanism. The authors remark, "Whether or not this time difference is related to the magnitude, depth, or any other parameter of the EQ cannot be ascertained from the small sample we have at hand". Based on our small experience from the three destructive Greek continental EQs, we suggest that the larger the variance of spatial distribution of local strengths in the focal zone is, the longer the duration of the EM gap (Eftaxias et al., 2000, 2001).

It is remarkable that precursory DC-ultra low frequency (ULF) signals have been also reported on April 18 and April 19, 1995 for the Kozani EQ and a prediction was issued by the VAN group on April 30, 1995 (Varotsos et al., 1996). The recorded VLF-VHF signals were detected within the time window of the VAN prediction (Eftaxias et al., 2000).

An EQ is a long-term complex stress accumulation and release process occurring in the highly heterogeneous Earth's crust and mantle. The various stages of EQ preparation are accompanied by EM emissions at different frequency bands ranging from ULF to VHF.

In each of the EQ preparation stages the signals emitted are located into "particular" rather narrow frequency regions. As a result, various research groups record EM emissions at narrow, specific frequency bands, attempting to relate them with an emission mechanism and, if possible, with the different stages of the EQ preparation. The researchers follow this strategy not only in the geophysical (Diodati et al., 1991) but also in the laboratory scale (e.g. Ohnaka and Mogi, 1982; Petri et al., 1994; Garcimartin et al., 1997; Carrillo et al., 1998).

Based on the above observations, it is evident that the selection and study of specific, rather narrow frequency bands is important and meaningful, allowing detailed study of different phases of the EQ preparation. Our group attempts to detect EM signatures corresponding to the last phase of the EQ preparation process, e.g. the nucleation phase. Therefore, we have decided to study EMA in the VLF $(3 \mathrm{kHz}$, $10 \mathrm{kHz}$ ) and VHF (41 MHz and $54 \mathrm{MHz}$ ) frequency bands based on (i) rock fracturing experiments (e.g. Ohnaka and Mogi, 1982; Carrillo et al., 1998) (ii) the very short time lag between VLF-VHF precursory EM signals and the EQs.

Mainly, in this paper we deal with the VHF (41 MHz) pre-seismic EMA (see Fig. 2(a)). We note that the wavelet spectra of the anomalies at $41 \mathrm{MHz}$ and $54 \mathrm{MHz}$ are morphologically very similar during their whole recording time (Eftaxias et al., 2002a). There are basic reasons for this choice. This EM emission have a rather long duration (sampled at $1 \mathrm{~Hz}$ ), thus it provides sufficient data for analysis. The emission ceases almost one hour before the EQ, i.e. is very close to a possible critical point, therefore a suitable theory can be applied to investigate this critical behavior. In the case of this shallow EQ clear surface fault traces of 8-12 km in length were observed (Meyer et al., 1998), consequently it is expected that the $\mathrm{MHz}$ signal escaped without significant degradation of its spectral characteristics. 

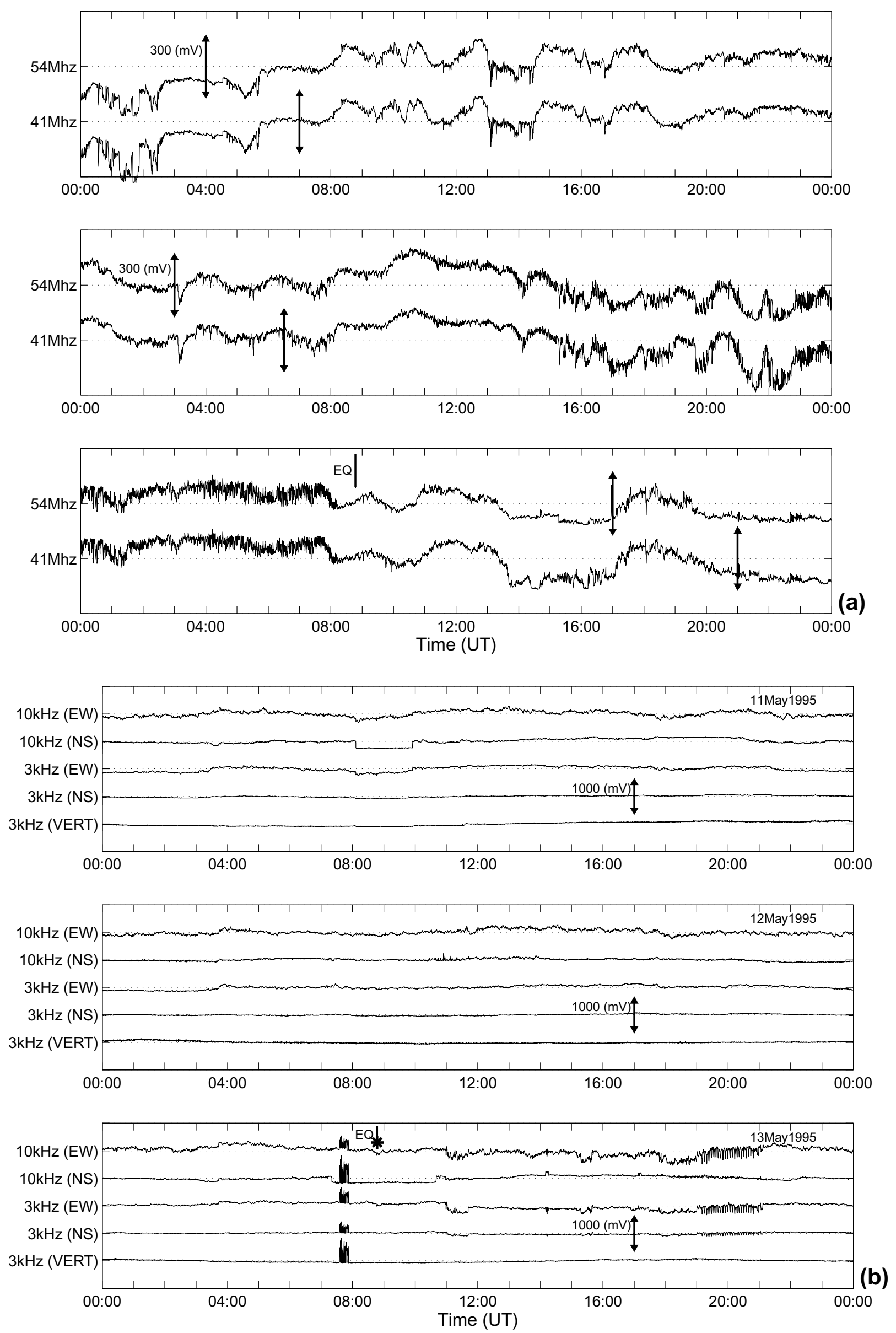

Fig. 2. Electromagnetic time-series averaged over 20 seconds at Zante station at (a) 41 and $54 \mathrm{MHz}$ and (b) at 3 and $10 \mathrm{kHz}$. The asterisk indicates the time of the EQ occurrence. 
It is known that there is no consensus on the physical mechanism of VLF-VHF signal's emission (Fujinawa et al., 1997 and references therein; Gershenzon and Bambakidis, 2001 and references there in). Many authors suggest that EM waves are directly emitted from the focal region (e.g. Warwick et al., 1982; Guo et al., 1994; Yoshino and Sato, 1994). We note the recently suggested relevant approach "Plasmon-decay model for origin of EM wave noises in the EQs" (Kamogawa and Ohtsuki, 1999a,b; Tanaka et al., 1999). We remind that the time evolution of the precursory EMAs associated with the Kozani-Grevena EQ reveals similarities to that observed in laboratory acoustic and EM emissions during different stages of failure preparation process in rocks. If we consider that the same dynamics govern the large-scale EQ and the laboratory scale sample rheological structure, the results of this analysis lead to the suggestion that the recorded anomalies are probably emitted from the focal area during micro-fracturing process (Eftaxias et al., 2002a). This suggestion is also supported by recent experimental field data from the Athens EQ where the precursory $\mathrm{EM}$ anomalies included two clear signals at the $\mathrm{kHz}$ band with an energy ratio (second signal to first) $\sim 5$ and the radar interferometry analysis showed two separate faults with an energy ratio (second to first) of 5 (Eftaxias et al., 2001). In any case, detailed discussion of the emission mechanism for the VLF-VHF signals we observe in conjunction with EQs is outside the scope of this report. However, according to our opinion, one might suggest the following scenario: In the case of Kozani-Grevena EQ, the pre-seismic processes in the focal zone covered a surface zone approximately of the same size. Several laboratory experiments (e.g., Enomoto and Hasimoto, 1990; Enomoto et al., 1994) have shown that exo-electrons and changed particles are emitted from rocks during micro-fracturing; this emission is of the order of $1 \mathrm{C} / \mathrm{s}$ for rocks with dimensions of a few meters. Such emissions of charges from stressed rocks around the focal area accompanying micro fracturing, can act as a current system in a conductor with a length of the order of the subsequent fracture generated by the EQ (Biagi, 1999). In general, the radiation pattern of an antenna can be effectively excited, only by certain frequencies corresponding to the characteristic length scales of the antenna, i.e., $k L \sim 1$, where $k$ denotes the radiation's wavenumber and $L$ represents the fault's scale length. For the Kozani-Grevena EQ, the (final) spatial scale $L$ is of the order of $10 \mathrm{~km}$ (Meyer et al., 1998). Consequently, the corresponding spectral content of the pre-seismic EM activity has finally been extended to lower frequencies (i.e., a few $\mathrm{kHz})$ whereas at intermediate stages $(L<10 \mathrm{~km})$ of the fault's creation, the expected EM activity was located in higher frequencies. The fact that the 3 and $10 \mathrm{kHz}$ preseismic EMAs were detected at the end of the 41 and $54 \mathrm{MHz}$ pre-seismic emissions supports the above proposed scenario.

\section{Experimental Evidence for Critical Dynamics in Fracture Process}

The increased interest in real materials in condensedmatter physics has brought disordered systems into spotlight. Heterogeneity changes the free-energy landscape of a system, and can introduce metastable states with large energy barriers. On long length scales and practical time scales, a system driven by an external field will move from one metastable local free-energy minimum to the next while emitting energy. The motion from one local minimum to the next is a collective process involving many local domains in a local region. Hence, the fracture in these heterogeneous systems is usually preceded by precursors in the form of avalanches of varying amplitude (or energy), duration, and time interval between bursts. In the frame of this consideration, the detected series of EM fluctuations, which emerged in the VHF frequency band during the last $\sim 30$ hours before the EQ, might reflect the EM response of the energy rearrangements that occur in the pre-focal area as the system evolution proceeds through jumps from one metastable state to another metastable state. The critical state is characterized by precursors - pulses with power law spatial and temporal distribution functions limited only by the size of the system. Thus, in the study of fracture in terms of criticality we are interested in determining the statistics of the series of the recorded EM pulses (with respect to their amplitude, energy, duration or time interval between two successive bursts) with no regard to their spectral composition (e.g. Diodati et al., 1991; Cannelli et al., 1993; Petri et al., 1994; Zapperi et al., 1997; Carrilo, 1998; Bofetta et al., 1999). From the detected signals we obtain both, the time-series of the EM fluctuation amplitudes and the time-series of the waiting times, i.e. the time intervals between two successive fluctuations. We argue that statistical analysis of these two time-series indicates an underlying critical dynamics.

\subsection{Estimating the power spectrum scaling exponent}

Initially, we focus on the statistics of the detected series of EM fluctuations with respect to their amplitude, let's say $A\left(t_{i}\right)$. We attempt to investigate autocorrelation structures in this time-series. Any time-series may exhibit a variety of autocorrelation structures; successive terms may show strong (brown noise), moderate (pink noise) or no (white noise) positive correlation with previous terms. The strength of these correlations provides useful information about the inherent "memory" of the system. The power spectrum $S(f)$, the Fourier transform of the autocorrelation function $C_{A}(\tau)=$ $\langle A(t) A(t-\tau)\rangle$, is probably the most commonly used technique to detect structure in time-series. Although the power spectrum is only the lowest order statistical measure of the deviations of the random density field from homogeneity, it directly reflects the physical scales of the processes that affect structure formation. If the time-series $A\left(t_{i}\right)$ is a temporal fractal then a power law spectrum of the recorded time-series is expected:

$$
S(f)=\alpha \cdot f^{-\beta}
$$

where the power spectrum $S(f)$ quantifies the correlations at the time scale $\tau \sim 1 / f$ and $f$ is the frequency of the Fourier transform. In a $\log S(f)-\log f$ representation the power spectrum is a straight line, with $\beta$ constituting its linear spectral slope. The spectral amplification $\alpha$ quantifies the power of the spectral components following the power spectral density law. Applying the least square method, we calculated the spectral slope $\beta$, the spectral amplification $\alpha$, and the linear correlation coefficient $r$ of the power law fit, dividing the signal into successive segments of 1024 samples each, in order to study their time evolution. The Continu- 

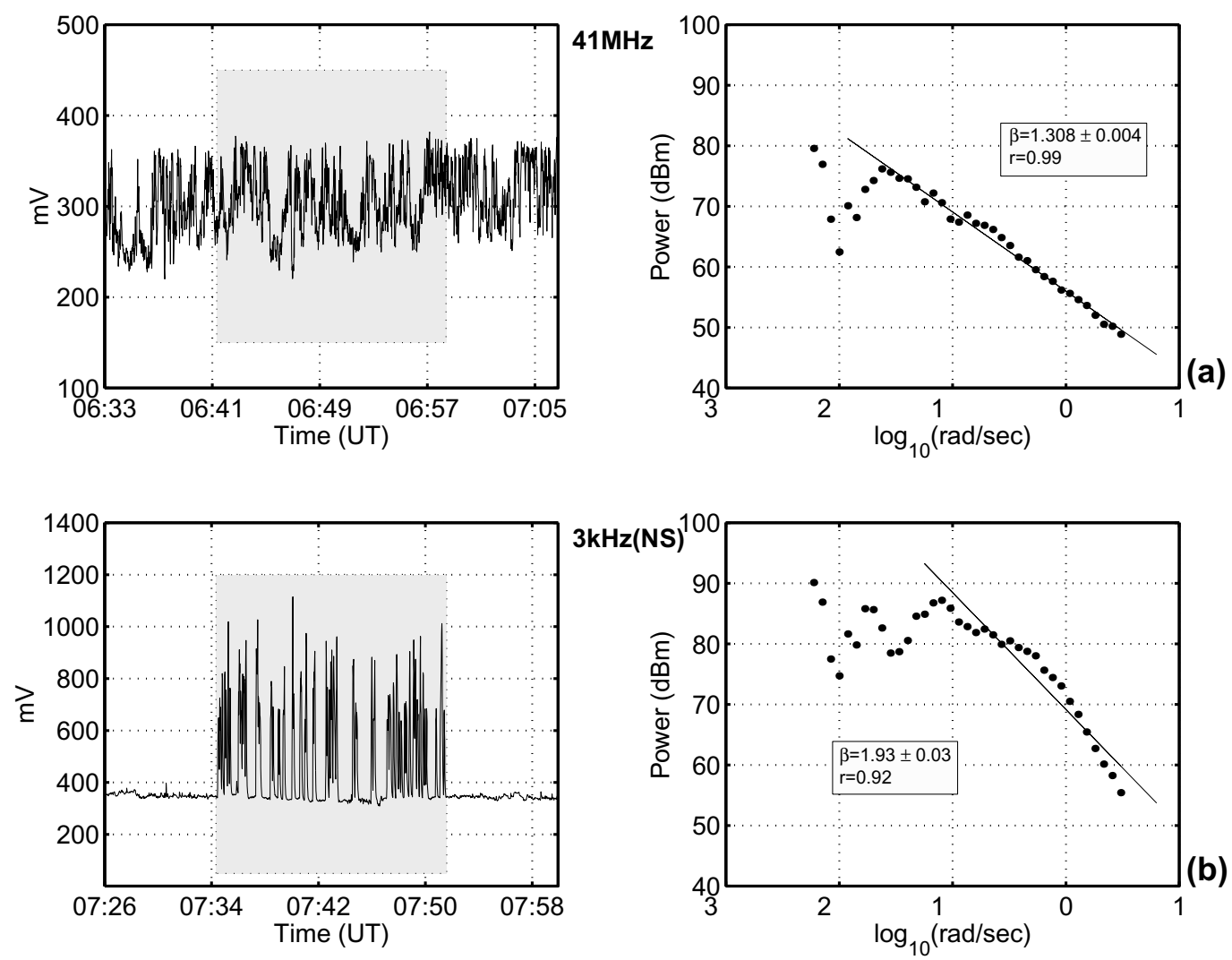

Fig. 3. Fitting of Eq. (1) i.e. of power spectral density $S(f)$ versus frequency, obtained (a) from a raw data sample of the $41 \mathrm{MHz}$ EMA and (b) from the raw data of the detected (NS) $3 \mathrm{kHz}$ clear anomaly as presented in Fig. 2(b).

ous Wavelet Transform (CWT), using Morlet wavelet, was used, since being superior to the Fourier spectral analysis providing excellent decompositions of even transient, nonstationary signals (Kaiser, 1994). Our approach is to calculate the parameters $\alpha, \beta, r$ dividing the signal into successive segments of 1024 samples each, in order to study not only the presence of a power law but also the time evolution of the associated parameters $\alpha, \beta, r$. The data fit the power law quite well. In Fig. 3(a) we show an example of fitting of Eq. (1), i.e. of power spectral density versus frequency plotted in log-log scales. This finding suggests that the time-series under study is governed by scaling lows. This implies that during the EMA each value co-varies not only with its most recent value but also with its long-term history in a scale invariance, fractal manner; the system refers to its history in order to define its future (non-Markovian behavior). From another viewpoint the finding of $1 / f$ behavior is a reflection of the fact that the final output of fracture is affected by many processes that act on different time scales. The abovementioned results are in good agreement with the relevant prediction based on the hypothesis that the evolution of the Earth's crust toward the (general) failure may take place as a critical phenomenon not only in a seismological sense but also in the EM sense.

Concerning the evolution of the slope $\beta$ (Fig. 4(b)) one can recognize the significant decrease of its fluctuation with the time in the narrow range $\beta=1.5 \pm 0.2$ during the last few hours, almost 8 hours, of the pre-seismic period. All the theories and models of precursory phenomena predict that stress and strain accumulation should become non-linear at the end of the loading cycle, producing rapidly accelerating effects. Thus, we can expect the dominance of the spectral characteristics of the pre-seismic EM activity over those of the EM background just before the EQ. In this spirit, we constructed (see Fig. 4(c)) a fence of intervals with $\beta=$ $1.5 \pm 0.2$ (gray stripes). We observe a progressive increase of the time intervals with $\beta=1.5 \pm 0.2$ as approaching the EQ. The spectral slope $\beta$ exhibits large random fluctuations out from the range $\beta=1.5 \pm 0.2$ just after the cessation of the signal, almost 1 hour prior to the EQ. This behavior may constitute signature of an underlying precursory critical dynamics.

The linear correlation coefficient $r$ is a measure of the goodness of fit to the power law (1). The temporal evolution of $r$ (Fig. 4(d)) reveals that at the tail of the pre-seismic time-series the fit to the power law was continuously excellent $(r>0.98)$. In light of this behaviour, we construct a new fence, considering intervals (gray stripes) with $\beta=1.5 \pm 0.2$ and $r>0.98$. One can recognize a progressive increase of the time intervals with $\beta=1.5 \pm 0.2$ and $r>0.98$ approaching the EQ. It is remarkable that this behavior breaks down just after the cessation of the signal. The combined evolution of the parameters $\beta$ and $r$ may represent an enhanced fingerprint for the organization of the system towards the critical point as coded in the power law. 

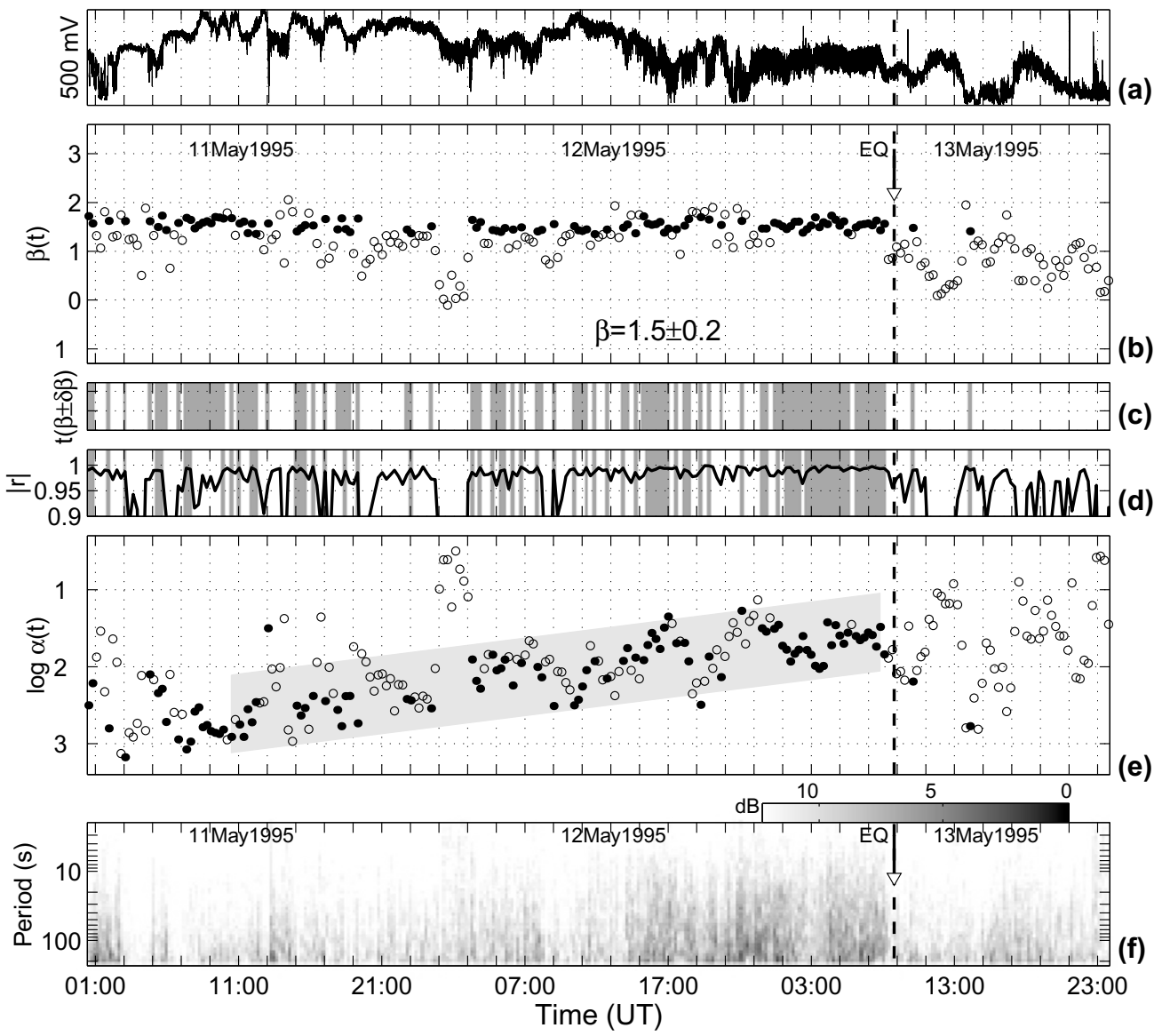

Fig. 4. (a) The $41 \mathrm{MHz}$ EMA recorded prior to the May 13, 1995 Kozani-Grevena EQ (the event is indicated with a vertical dashed line in all the panels); (b) the corresponding spectral slope $\beta$. Solid circles correspond to segments with $\beta$ values equal to $1.5 \pm 0.2$, while empty circles to segments with $\beta$ values outside this range; (c) gray zones correspond to $\beta$ values of $1.5 \pm 0.2$; (d) the variation of linear correlation coefficient $r$ of the power law spectral fitting (solid line). Grey zones correspond to $\beta$ values of $1.5 \pm 0.2$ and $r$ greater than 0.98 ; (e) time-dependence of the spectral amplification parameter $\alpha$. The grey zone corresponds to the interval of exponential increase of $\alpha$; (f) the wavelet spectral amplitude evolution of the signal. The analysis has been performed on raw data (sampled at $1 \mathrm{~Hz}$ ).

\subsection{Estimating the distribution function of the time in-} tervals between two successive bursts

Now, we study the distribution function of the laminar or waiting times. i.e., the time intervals $l$ between two successive bursts larger than the noise threshold. Recently, Contoyiannis et al. (2002a) have presented a new method for the study of the critical fluctuations. They argue that the fluctuations in a complex system at the critical point can be described in terms of intermittent dynamics of type I. This method has been applied to the VHF time-series under study (Contoyiannis et al., 2002a). More precisely, in the frame of this approach, Contoyiannis et al. (2002b) found that the part of the signal between May 12 15:00 (UT) and May 12 21:15, possesses a remarkable stationarity. This finding meets the relevant theoretical expectation. This behavior is a signature of a continuous phase transition (Contoyiannis and Diakonos, 2000; Contoyiannis et al., 2002a). The theory further predicts that the distribution of laminar lengths in the above mentioned window of stationarity follows a power law $P(l) \sim l^{-z /(z-1)}$, where the parameter $z$ represents the universality class. The analysis of the experimental data reveals that the distribution of the laminar lengths actually follows a power law within the above-mentioned window; the associated critical exponent has been found $-z /(z-1)=-1.20$
(Fig. 5). This evidence is an independent confirmation that the sequence of the precursory EM events is a temporal fractal.

The critical value $z=6$ classifies the reported critical transition to the 3D-Ising-universality class. Moreover, the observed value of the characteristic parameter $z$, which is shown to be related to the effective intermittent dynamics at the critical point (Contoyiannis and Diakonos, 2000; Contoyiannis et al., 2002a) leads through theory (Schuster, 1989) to the prediction that the power law of the critical fluctuations should exhibit a power law of the form $S(f)=$ $\alpha \cdot f^{-\beta}$ (2) with $\beta=1.4$. The present independent statistical analysis reveals that the $\beta$ values oscillate around 1.4 in the same window. The perfect agreement between the two independent analysis methods, increase the confidence in the reliability of our conclusion.

\subsection{Indication of a general scale invariance of the frac- turing process}

Special attention has to be drawn to the following point: Diodati et al. (1991) have presented evidence of criticality in volcanic rock ruptures associated with the Strobolian activity, measured by acoustic emissions (AE). The distribution of the time intervals $l$ between two successive AE bursts exhibits a power law behavior $P(l) \sim l^{-1.20}$. We remind that 


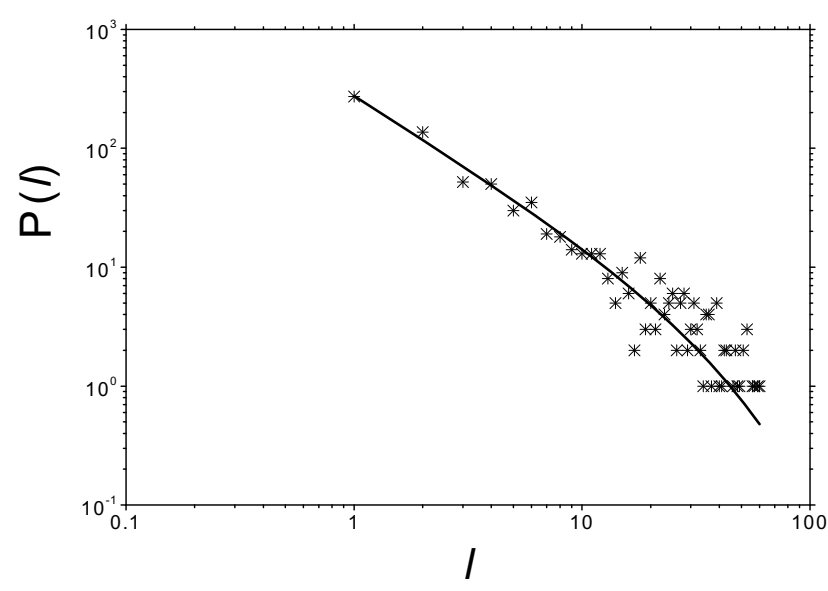

Fig. 5. Distribution of the EM fluctuations from May 12 15:00 (UT) up to May 12 21:15 with respect to their time separation.

the distribution of the time intervals $l$ between two successive EM bursts in our data also exhibits the power law behavior $P(l) \sim l^{-1.20}$. The perfect agreement between the two scaling exponents increases the confidence in the reliability of our conclusion that the detected sequence of EM bursts is a sub-product of the fracture process in the pre-focal area. Moreover this agreement indicates that there is a general scale invariance of the fracturing process.

In summary, the above-mentioned findings indicate that $\sim 30$ hours prior to the Kozani-Grevena EQ a new stage of the EQ preparation process was emerged; the associated mechanism appears characteristics of criticality.

\subsection{Estimating power spectrum scaling exponent on VLF precursors}

As we mentioned in Section 2, we mainly focus on the statistical analysis of the VHF anomaly due to its rather long duration. If the detected signals are related with an underlying critical mechano-EM mechanism, a power law should be also valid for the VLF anomalies. Unfortunately, due to the short duration of the $\mathrm{kHz}$ anomaly, we are not able to inspect the parameters' evolution as in the case of the $\mathrm{MHz}$ anomalies. In Fig. 3(b), we show the fitting of Eq. (1) plotted in log-log scale for the $3 \mathrm{kHz}$ precursory EMAs presented in Fig. 2(b). The corresponding power law parameters are: $r=0.92$ and $\beta=1.93 \pm 0.03$. This scaling property indicates that almost 1.5 hour prior to the Kozani-Grevena EQ (at the tail of the precursory sequence of VHF EM bursts) a new phase of fracture mechanism was abruptly emerged with critical behavior; this mechanism emits avalanches in the VLF frequency band. It should be noticed that the study of the precursory VLF-fluctuations associated with the Athens, Greece, EQ of 7 September 1999 (Eftaxias et al., 2000; Eftaxias et al., 2001) also reveals power law behavior (Eftaxias et al., 2002b).

\subsection{Comments concerning the treatment of the experi-} mental data

At this point we would like to add a few comments concerning the treatment of our data. The fractal curves have the following important characteristic: they look the same whatever the scale of observation within the scale range of validity for relation (1). This introduces the concept of scale

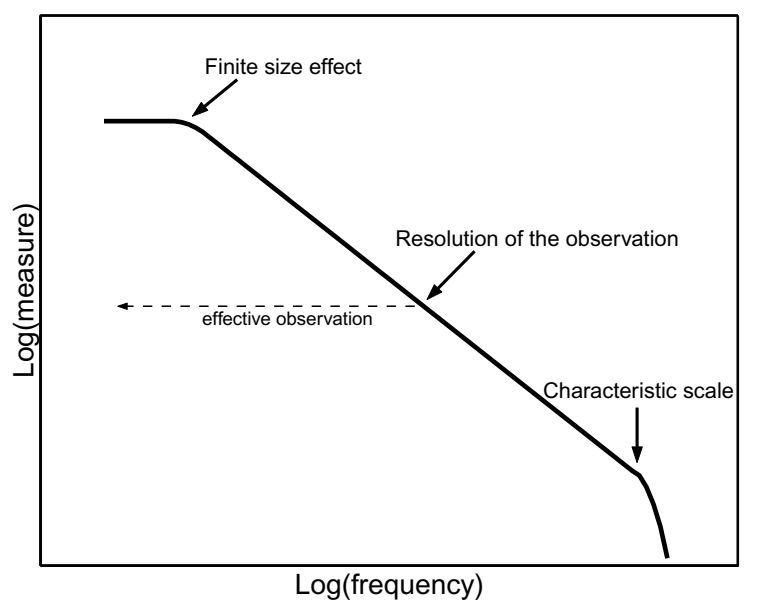

Fig. 6. Scale invariance of a fractal set or measure. Scale invariance is expected by a linear plot in a log-log frame. For natural fractals, scale invariance is bounded towards small scales (large frequencies) by an elementary scale or by the resolution of the analysis, and towards large scales (small frequencies) by a finite size effect.

invariance. However, for natural (or physical) fractals, relation (1) holds only within a limited range of scale. The upper frequency bound generally reveals a characteristic scale possibly associated to the physical process leading to fractal geometry, or it is simply the result of the resolution of the observation, whereas the lower bound frequency can be associated with the finite size of the object under consideration (Weiss, 2001). The plot corresponding to data with an $1 \mathrm{~Hz}$ sampling rate (Fig. 3) clearly shows that the upper bound in the under study case is of the order of a few tens of seconds. This evidence implies that if we know the behavior of the temporal evolution of EM fluctuations provides information about the behavior of the emission up to some tens of seconds later. The sampling rate $(1 \mathrm{~Hz})$ is higher in comparison with the observed lower frequency bound and thus this sampling rate captures the fractal pattern of the under study time-series. Lowering the sampling rate has the effect of excluding from the analysis the smallest scales: this implies that the estimated fractal dimension may not be appreciably affected provided the same kind of fractal structure is found in the scales still present. Further increasing this sampling rate above $1 \mathrm{~Hz}$ does not add any additional relevant information. In general, any real data comprise a sample from some unknown population. In choosing a model to fit these data a prediction of the nature of the population is made. If the data fit the model then the prediction is supported. If the data do not fit, then either the model is wrong, or the sample is biased (Pickering et al., 1995). As our data fit the power law quite well (Fig. 3) it might be concluded that the data are almost certainly taken from a power law.

\section{Further Arguments Supporting the Critical Point Hypothesis}

The above-mentioned findings might not be sufficient to characterize the reported pre-seismic EM phenomenon as the output of a critical system. Further arguments are needed to support this hypothesis. 


\subsection{Argument I}

It has been suggested that the rate of Benioff strain (the square root of seismic energy) release accelerates prior to the occurrence of the large EQ (Sornette, 2000). On the other hand, many researchers have proposed that the precursory EM activity could be considered as a by-product of the foreshock activity in the EM domain (Kanamori, 1996; Molchanov, 1999; Hayakawa, 1999; Gershenzon and Bambakidis, 2001; Telesca et al., 2001). This assumption is further strengthened by the surprising correlation found in the energy domain between the two strong pre-seismic $\mathrm{kHz}$ EM signals and the two faults of the September 9, 1999 Athens EQ (Eftaxias et al., 2001). In this case, two clear $\mathrm{kHz}$ EM signals have been detected before the Athens EQ with the first signal containing approximately the $20 \%$ and the second the $80 \%$ of the total escaped EM energy. The fault modelling of Athens EQ, based on information obtained by radar interferometry, showed two faults. The main fault segment is responsible for the $80 \%$ of the total seismic energy released while the secondary fault segment for the remaining $20 \%$. We note that a recent seismic data analysis carried out by M. Kikuchi, using the now standard methodology (Kikuchi and Kanamori, 1990) supports the hypothesis that a two-events solution for Athens EQ is more likely than a single event solution (Eftaxias et al., 2001). According to Kikuchi, there was probably a subsequent $(M w=5.5) \mathrm{EQ}$ after about $3.5 \mathrm{~s}$ of the main event $(M w=5.8)$. Additionally, a series of laboratory experiments show a direct correlation between VLF-VHF acoustic (foreshocks) and EM activity due to the microfracturing in rocks under increasing stress. Hadjicontis and Mavromatou (1996), who performed laboratory experiments in the frequency range from some $\mathrm{kHz}$ to some $\mathrm{MHz}$, found that the initiation of the accelerating uncontrolled EM emission, which necessarily leads to failure, is related to a mechanical load of about the $95 \%$ of the fracture strength. Building upon these concepts we suggest that the presence of accelerating rate in the pre-seismic EM activity may provide another indication of an underlying critical mechanism.

The power law spectrum (1) is characterized, in addition to the slope $\beta$, by the spectral amplification $\alpha$, measuring the instant intensity of the EM source. The evolution of $\alpha$ is shown in Fig. 4(e). We observe that $\alpha$ systematically increases, nearly exponential, during the last few tens of hours before the EQ. The rate of increase is found comparable to the overall duration of the signal. This continuous increase in the signal's intensity approaching the event can be also seen in the wavelet scalogram (Fig. 4(f)) that visualises the evolution of the amplitude of sequence of pulses constituting the pre-seismic EM signal. Consequently, the expected signature, i.e. the acceleration of the emission rate approaching the EQ, was indeed observed before the Kozani-Grevena EQ. This result strongly supported by the emergence of the strong $\mathrm{kHz}$ multi-peaked signals (with duration almost 30 $\min$ ) at the tail of the detected MHz pre-seismic EMA.

\subsection{Argument II}

Mavromatou-Hadjiconti (1995) reported a characteristic shift of the frequency content of the EM emission to lower frequencies approaching the failure. It is important to note that this behavior is in full agreement with the observed shift (from $\mathrm{MHz}$ to $\mathrm{kHz}$ ) of the acoustic activity during the prefailure stage ( $90 \sim 100 \%$ of the failure strength) in Ohnaka and Mogi (1982)'s classical laboratory experiment. On the other hand, the extended Chinese field experience (Qian et al., 1994) for this type of EM emissions, based on a large number of observations, shows that the pre-seismic anomalies are detected earlier in the VHF than in the VLF frequency bands. Many workers have reported that the number of VLF pulse-like signals has been known to increase clearly just before EQs (Fujinawa, et al., 1997, and references there in). Based on these findings, we suggest that the shift of the frequency content of the EM emission to lower frequencies (from $\mathrm{MHz}$ to $\mathrm{kHz}$ ) may provide an additional indicator of an underlying critical dynamics. Such shift of the frequency content was also observed before the Kozani-Grevena EQ; it is reminiscent that strong $3 \mathrm{kHz}-10 \mathrm{kHz}$ signals have been detected at the tail of the $41 \mathrm{MHz}-54 \mathrm{MHz}$ anomalies.

\subsection{Argument III}

The observation of foreshocks is the most convincing evidence that there is a nucleation stage before major EQs. The Kozani-Grevena EQ was preceded, within 30 min of the main shock, by an unusual foreshocks activity, i.e., by five foreshocks with $M>3.5$ clustered within $2 \mathrm{~km}$ of one another, about 5 to $10 \mathrm{~km}$ from the main shock epicentre (Bernard et al., 1997). This foreshock activity reported some minutes after the cessation of the pre-seismic EMAs. This evidence supports the idea that the detected EMAs could be considered as a by-product of the foreshock activity. The unusual foreshock activity for the a-seismic Kozani-Grevena region may be due to rupture of asperities in zone associated with the nucleation phase of the main rupture and thus constitutes another independent indicator of the underlying critical mechanism.

In our opinion, the time evolution of the parameters $\alpha, \beta$ and $r$ is in agreement with characteristic feature of the nucleation phase in the pre-focal area. The stability of the spectral slope $\beta$ indicates that the fracture mechanism retains its identity as a system that is organized towards a critical point, while, the continuous increase of amplification $\alpha$ is compatible with the idea that the underlying emission mechanism is spatially extending.

\subsection{The hypothesis of a fractal "geo-antenna"}

The principle feature of criticality is the fractal organisation both in space and time. Thus, the evolution of the Earth's crust towards the critical point could involve the formation of a fractal structure in the focal zone (Hayakawa et al., 1999). Fragment size distribution was widely investigated, due to each importance for the understanding of fragmentation processes. There is strong evidence for the fractal character of size distributions: the lack of a "characteristic size" is confirmed for a large variety of geomaterials and experimental conditions (Turcotte, 1993). The above concepts could lead someone to extend the idea proposed in Section 3 as a probable scenario and support that the "antenna" could be considered as a fractal "geo-antenna". Note that fractal antennae have very special properties. (i) they are broad band antennae, i.e., they radiate very efficiently for a wide range of frequencies and this frequency range is specified by the smallest and largest size present in the antenna and (ii) they can display considerable gain over normal dipole type of antennae. 
The detected sequence of EMAs (from $\mathrm{kHz}$ up to $\mathrm{MHz}$ ) is compatible with the hypothesis of the presence of a fractal "geo-antenna".

\section{Discussion and Conclusions}

Various field observations seem to agree qualitatively with the idea that the signals of the DC-ULF band tend to appear earlier (Gokhberg et al., 1995; Qian et al., 1994; Uyeda, 1996; Varotsos et al., 1996; Hayakawa and Fujinawa, 1994; Hayakawa, 1999). A time period of 1-2 months or more is usually elapsed between the detection of the DC-ULF preseismic EM activity and the occurrence of strong EQ. The time lag between the initiation of VLF-VHF signals and EQs is of the order from a few tens of hours to a few of hours.

In the following, we shall review cases with evident DCULF EM hallmark of critical dynamics up to a few months before the EQ: (a) ULF EM emissions associated with a large EQ $M_{w}=8.2$ occurred at Biak Island, on February 17,1996 , have been analysed on the basis of the selforganized criticality concept by Hayakawa et al. (2000). The slope $\beta$ was found to fluctuate in the range from 2.0 to 0.7 . They found that the spectrum of emissions exhibited power law behaviour (1) with $\beta \sim 1.0$ for about two weeks (Dec. $23 \sim$ Jan. 10), that is, about 1.9-1.5 months before the EQ. (b) Hayakawa et al. (1999) reported the presence of seismogenic ULF emissions associated with the Guam $\left(M_{S}=8.0\right)$ EQ on August 8, 1993. They found that the spectrum of emissions exhibited power law behaviour, with slope $\beta$ fluctuating in the range from 2.5 to 0.7 . The authors, taking into account the value of the slope $\beta=1.2$ as a threshold, found a gradual increase of the periods with smaller $\beta<1.2$ towards the EQ date and suggested that this increase can be considered as a candidate for precursory signature. (c) Telesca et al. (2001) have studied ULF geoelectrical signals associated with the seismicity of the Irpinia-Basilicata area (southern Italy). Their spectral analysis led to values of slope $\beta$ varying from 1.12 to 1.78 with average $\sim 1.4$. They observe a tendency of the critical exponent $\beta$ to converge toward unity about 200 days before the M 4.5 April 3, 1996, EQ. The authors suggested that this behaviour is indicative of underlying self-organized criticality. These findings support the hypothesis that the evolution of the Earth's crust toward the critical point takes place not only in a seismological sense but also in the ULF EM sense.

An important observation is that in all the abovementioned cases a tendency of the ULF EM critical exponent $\beta$ to converge toward $1.5 \pm 0.2$ just before the EQ is evident. This behavior is in quantitative agreement with the reported in this study evolution of the VHF EM critical exponent $\beta$. The concept that this value of critical exponent $\beta$ might be common feature of the EQ nucleation process cannot be excluded.

In summary, we found that the spectrum of the precursory VHF (41 MHz) EM emission, during the last few tens of hours before the Kozani-Grevena EQ, exhibited time intervals of power law (1) with the following unique (in contrast to the background noise) characteristics: (i) Stable spectral exponent $\beta$, close to 1.5. (ii) Excellent linear correlation coefficient $r>0.98$. It is remarkable that the number of the time intervals meeting (i) and (ii) progressively increases approaching the moment of the EQ; at the tail of the preseismic time-series the spectral parameters exhibited continuously the values $\beta=1.5 \pm 0.2$ and $r>0.98$. Moreover, the detected EM activity appeared a systematic, nearly exponentially, increasing amplification $\alpha$, i.e. an accelerating EM energy release, during the last few tens of hours before the EQ. Finally, the detected sequence of EM signals appeared a clear frequency shift from $\mathrm{MHz}$ to $\mathrm{kHz}$ at the end of the detected sequence of EMAs.

All these findings support the hypothesis that the evolution of the Earth's crust toward the critical point may take place not only in the ULF-EM sense but also in the VHF-EM sense. This hypothesis is strengthened from the observed unusual foreshocks activity associated with the Kozani-Grevena EQ almost some minutes after the cessation of the pre-seismic anomalies and some minutes before the EQ.

All of these concepts remain to be investigated further. But if this behaviour of the ULF and VHF signals could be confirmed, a study devoted to EQ preparation process should have to make predictions consistent with the reported critical features of both the ULF signals, a few months before the $\mathrm{EQ}$, and VHF signals, just before the EQ.

Acknowledgments. This work was supported by the Special Research Account of the University of Athenes (grant no. 70/4/5847). We acknowledge that the comments by Y. Tanaka and another referee were helpful in improving the present work.

\section{References}

Asada, T., H. Baba, M. Kawazoe, and M. Suguira, An attempt to delineate very low frequency electromagnetic signals associated with earthquakes, Earth Planets Space, 53(1), 55-62, 2001

Bernard, P., P. Pinettes, P. Hadjidimitriou, E. Scordilis, G. Veis, and P. Milas, From precursors to predictions: a few recent cases for Greece, Geophys. J. Int., 131, 467-477, 1997.

Biagi, P., Seismic effects on LF radiowaves, in Atmospheric and Ionospheric Electromagnetic Phenomena associated with Earthquakes, edited by M. Hayakawa, pp. 535-542, Terrapub, Tokyo, 1999.

Boffetta, G., V. Carbone, P. Giuliani, P. Veltri, and A. Vulpiani, Power laws in solar flares: self-organized criticality or turbulence?, Phys. Rev. Lett., 83, 4662-4665, 1999.

Cannelli, G., R. Cantelli, and F. Cordero, Self-organized criticality of the fracture processes associated with hydrogen precipitation in niobium by acoustic emission, Phys. Rev. Lett., 70, 3923-3926, 1993.

Carrillo, L., L. Manosa, J. Ortin, A. Planes, and E. Vives, Experimental evidence for universality of acoustic emission avalance distributions during structrural transitions, Phys. Rev. Lett., 81, 1889-1892, 1998.

Contoyiannis, Y. and F. Diakonos, Criticality and intermittency in order parameter space, Phys. Lett. A, 268, 286-292, 2000.

Contoyiannis, Y., F. Diakonos, and A. Malakis, Intermittent dynamics of critical fluctuations, Phys. Rev. Lett., 89, 35701/1-35701/4, 2002a.

Contoyiannis, Y., F. Diakonos, P. Kapiris, A. Peratzakis, and K. Eftaxias, Fingerprints of intermittent and critical behaviour of pending earthquake in electromagnetic anomalies, EGS XXVII General assemply, NHO62, $2002 b$.

Diodati, P., F. Marchesoni, and S. Piazza, Acoustic emission from volcanic rocks: an example of self-organized criticality, Phys. Rev. Lett., 67, 22392243, 1991.

Guo, Z., B. Liu, and Y. Wang, Mechanism of electromagnetic emissions associated with microscopic and macroscopic cracking in rocks, in Electromagnetic Phenomena Related to Earthquake Prediction, edited by M. Hayakawa and Y. Fujinawa, pp. 523-529, Terrapub, Tokyo, 1994.

Eftaxias, K., J. Kopanas, N. Bogris, P. Kapiris, G. Antonopoulos, and P. Varotsos, Detection of electromagnetic earthquake precursory signals in Greece, Proc. Japan Acad., 76(B), 45-50, 2000.

Eftaxias, K., P. Kapiris, J. Polygiannakis, N. Bogris, J. Kopanas, G. Antonopoulos, A. Peratzakis, and V. Hadjicontis, Signatures of pending earthquake from electromagnetic anomalies, Geophys. Res. Lett., 28, 
3321-3324, 2001

Eftaxias, K., P. Kapiris, E. Dologlou, J. Kopanas, N. Bogris, G. Antonopoulos, A. Peratzakis, and V. Hadjicontis, EM anomalies before the Kozan earthquake: A study of their behaviour through laboratory experiments, Geophys. Res. Lett., 29(8), 2002a.

Eftaxias, K., P. Kapiris, J. Polygiannakis, A. Peratzakis, J. Kopanas, G. Antonopoulos, and D. Rigas, Experience of short term earthquake precursors with VLF-VHF electromagnetic emissions, Natural Hazards and Earth System Sciences, 2002b (accepted).

Enomoto, Y. and H. Hashimoto, Emission of charged particles from indentation fracture of rocks, Nature, 346, 641-643, 1990.

Enomoto, Y., T. Shimamoto, A. Tsutsumi, and H. Hashimoto, Transient electric signals prior to rock fracturing: Potential use as an immediate earthquake precursor, in Electromagnetic Phenomena Related to Earthquake Prediction, edited by M. Hayakawa and Y. Fujinawa, pp. 253-259, Terrapub, Tokyo, 1994

Fujinawa, Y., K. Takahashi, T. Matsumoto, and N. Kawakami, Experiments to locate sources of earthquake-related VLF electromagnetic signals, Proc. Japan Acad., 73(B), 33-38, 1997.

Garcimartin, A., A. Guarino, L. Bellon, and S. Ciliberto, Statistical properties of fracture precursors, Phys. Rev. Lett., 79, 3202-3205, 1997.

Gershenzon, N. and G. Bambakidis, Modeling of seismo-electromagnetic phenomena, Russian Journal of Earth Sciences, 3, 247-275, 2001

Gokhberg, M., V. Morgunov, and O. Pokhotelov, Earthquake Prediction Seismo-Electromagnetic Phenomena, pp. 193, Gordon and Breach Publishers, Amsterdam, 1995.

Gershenzon, N. and G. Bambakidis, Modeling of seismo-electromagnetic Phenomena, Russian Journal of Earth Sciences, 3, 247-275, 2001

Hadjicontis, V. and C. Mavromatou, Laboratory Investigation of the Electric Signals Preceding earthquakes, in A Critical Review of VAN, edited by Sir J. Lighthill, pp. 105-117, World Scientific Publishing Co., Singapore, 1996.

Hayakawa, M., (ed.), Atmospheric and Ionospheric Electromagnetic Phe nomena Associated with Earthquakes, pp. 996, Terrapub, Tokyo, 1999.

Hayakawa, M. and Y. Fujinawa, (eds), Electromagnetic Phenomena Related to Earthquake Prediction, pp. 677, Terrapub, Tokyo, 1994.

Hayakawa, M., T. Ito, and N. Smirnova, Fractal analysis of ULF geomagnetic data associated with the Guam earthquake on August 8, 1993, Geophys. Res. Lett., 26, 2797-2800, 1999.

Hayakawa, M., T. Itoh, K. Hattori, and K. Yumoto, ULF electromagnetic precursors for an earthquake at Biak, Indonesia on February 17, 1996 , Geophys. Res. Lett., 27, 1531-1534, 2000.

Kaiser, G., A Friendly Guide to Wavelets, pp. 300, Birkhauser, 1994

Kamogawa, M. and Y.-H. Ohtsuki, Plasmon model for origin of earthquake related electromagnetic wave noises, Proc. Japan Acad., 75(B), 186-189, 1999 a.

Kamogawa, M. and Y.-H. Ohtsuki, Image-charge model for origin of electromagnetic wave noises in the earthquakes, in Atmospheric and Ionospheric Electromagnetic Phenomena Associated with Earthquakes edited by M. Hayakawa, pp. 401-404, Terrapub, Tokyo, 1999b.

Kanamori, H., A seismologist's view of VAN, in A Critical Review of VAN Earthquake Prediction from Seismic Electric Signals, edited by Sir J. Lighthill, pp. 339-346, World Scientific Publishing Co., Singapore, 1996.

Kikuchi, M. and H. Kanamori, Inversion of complex body waves, III, Bull. Seism. Soc. Am., 81, 2335-2350, 1990.

Mavromatou-Hadjiconti, C., Laboratory Investigation of the Electric Signals Preceding the Fracture of Crystalline Materials, Ph.D. Thesis, University of Athens, 1995.

Meyer, B., R. Armijo, D. Massonet, J. B. De Chabalier, C. Delacourt, J. C. Ruegg, J. Achache, and D. Papanastassiou, Results from combining tec- tonic observations and SAR interferometry for the 1995 Grevena earthquake: A summary, J. Geodynamics, 26, 255-259, 1998.

Molchanov, O., Fracturing as an underlying mechanism of seismo-electric signals, in Atmospheric and Ionospheric Electromagnetic Phenomena Associated with Earthquakes, edited by M. Hayakawa and Y. Fujinawa, pp. 349-356, Terrapub, Tokyo, 1999.

Nomikos, K. and F. Vallianatos, Electromagnetic variations associated with the seismicity of the frontal Hellenic arc, Geologica Carpathica, 49, 5760, 1998.

Ohnaka, M. and K. Mogi, Frequency characteristics of acoustic emission in rocks under uniaxial compression and its relation to the fracturing process to failure, J. Geophys. Res., 87, 3873-3884, 1982.

Petri, A., G. Paparo, A. Vespignani, A. Alippi, and M. Costantini, Experimental evidence for critical dynamics in microfracturing processes, Phys. Rev. Lett., 73, 3423-3426, 1994.

Pickering, G., J. Bull, and D. Sanderson, Sampling power-law distribution, Tectonophys., 248, 1-20, 1995.

Qian, S., J. Yian, H. Cao, S. Shi, Z. Lu, J. Li, and K. Ren, Results of observations on seismo-electromagnetic waves at two earthquake experimental areas in China, in Electromagnetic Phenomena Related to Earthquake Prediction, edited by M. Hayakawa and Y. Fujinawa, pp. 205-211, Terrapub, Tokyo, 1994.

Schuster, H., Deterministic Chaos, VCH Publishers, pp. 270, 1989.

Sornette, D., (ed.), Critical Phenomena in Natural Sciences, pp. 434, Springer, 2000.

Tanaka, H., M. Kamogawa, and Y. Ohtsuki, The interactions between bulk plasmons and electromagnetic waves assisted by surface roughness, Proc. Japan Acad., 75, 190-194, 1999.

Telesca, L., V. Cuomo, and V. Lapenna, A new approach to investigate the correlation between geoelectrical time fluctuations and earthquakes in a seismic area of southern Italy, Geophys. Res. Lett., 28(23), 4375-4378, 2001.

Turcotte, D., (ed.), Fractals and chaos in geology and geophysics, pp. 996 Cambridge University Press, 1993.

Uyeda, S., Introduction to the VAN method of earthquake prediction, in $A$ Critical Review of VAN-Earthquake Prediction from Seismic Electric Signals, edited by Sir James Lighthill, pp. 3-28, World Scientific Publishing Co., Singapore, 1996.

Vallianatos, F. and K. Nomikos, Seismogenic radioemissions as earthquake precursors in Greece, Phys. Chem. Earth, 23, 953-957, 1998.

Varotsos, P., M. Lazaridou, K. Eftaxias, G. Antonopoulos, J. Makris, and J. Kopanas, Short-term Earthquake Prediction in Greece by Seismic Electric Signals, in A Critical Review of VAN: Earthquake Prediction from Seismic Electric Signals, edited by Sir J. Lighthill, pp. 29-76, World Scientific Publishing Co., Singapore, 1996.

Warwick, J., C. Stoker, and T. Meyer, Radio emissions associated with rock fracture: Possible application to the great Chilean earthquake of May 22 1960, J. Geophys. Res., 87(B4), 2851-2859, 1982.

Weiss, J., Fracture and fragmentation of ice: a fractal analysis of scale invariance, Engineering Fracture Mechanics, 68, 1975-2012, 2001.

Yoshino, T. and H. Sato, The study of exciting process of seismogenic emissions at epicentre by magnetic flux based on the statistical analysis, in Electromagnetic Phenomena Related to Earthquake Prediction, edited by M. Hayakawa and Y. Fujinawa, pp. 631-640, Terrapub, Tokyo, 1994.

Zapperi, S., A. Vespignani, and H. Stanley, Plasticity and avalanche behaviour in microfracturing phenomena, Nature, 388, 658-660, 1997.

P. Kapiris, J. Polygiannakis, A. Peratzakis, K. Nomicos, and K. Eftaxias (e-mail: ceftax@phys.uoa.gr) 